\title{
A percepção dos cidadãos sobre as Infecções Sexualmente Transmissíveis (ISTs):
}

\section{Relato de experiência}

\author{
Citizens' perception of Sexually Transmitted Infections (STIs): Experience report \\ Percepción ciudadana de las Infecciones de Transmisión Sexual (ITS): Informe de experiencia
}

Recebido: 15/03/2021 | Revisado: 21/03/2021 |Aceito: 27/03/2021 | Publicado: 04/04/2021

Ianny Ferreira Raiol

ORCID: https://orcid.org/0000-0002-7776-723X Centro Universitário Metropolitano da Amazônia, Brasil

E-mail: raiolianny@hotmail.com

Marina Pereira Queiroz dos Santos

ORCID: https://orcid.org/0000-0002-2596-1241 Centro universitário Metropolitano da Amazônia, Brasil

E-mail: marinaqueirozsantos@hotmail.com

Jady Barreirinhas Barros

ORCID: https://orcid.org/0000-0002-8414-8744 Centro universitário Metropolitano da Amazônia, Brasil E-mail: jady_barros@hotmail.com

Carolyna Magno Corvello

ORCID: https://orcid.org/0000-0002-6395-0068 Centro Universitário Metropolitano da Amazônia, Brasil E-mail: carolmagno08@gmail.com

Adrielly Cristiny Mendonça Fonseca

ORCID: https://orcid.org/0000-0002-9706-6780 Centro universitário Metropolitano da Amazônia, Brasil E-mail: adriellycmf@gmail.com

Daniel Lucas Costa Monteiro

ORCID: https://orcid.org/0000-0003-0939-6120 Centro universitário Metropolitano da Amazônia, Brasil E-mail: daniellucas.k@gmail.com Shirley Aviz de Miranda

ORCID: https://orcid.org/0000-0001-7405-8369 Centro Universitário Metropolitano da Amazônia, Brasil E-mail: shirleyaviz@hotmail.com

\section{Resumo}

Objetivo: Relatar uma experiência vivenciada pelos autores do estudo em uma sala de espera de um Ambulatório em Belém do Pará, quanto a relação da percepção dos cidadãos acerca das Infecções Sexualmente Transmissíveis. Método: Explana um relato de experiência, realizado por acadêmicos de Enfermagem e Medicina no Ambulatório de Especialidade para os usuários que esperavam atendimento. A construção da atividade foi baseada nas etapas do Arco de Maguerez: I- Foi a elaboração da atividade no Ambulatório; II- Identificação dos pontos-chaves, possíveis vulnerabilidades e público-alvo; III- A teorização, investigação através de estudos bibliográficos; IV- Busca inovações tecnológicas referentes à problemática, e construção do material, evidenciando as principais ISTs; V- Realização da ação educativa. Resultados e Discussão: A atividade educativa proporcionou importantes diálogos sobre os conhecimentos de ISTs. Percebeu-se fragilidade quanto no conhecimento e por isso maior interesse sobre a transmissão das ISTs. Considerações Finais: Conclui-se ser fundamental o desenvolvimento de estratégias voltadas à saúde pública, com enfoque na prevenção, como a inclusão de ações visando qualificar o conhecimento sobre IST, utilizando táticas que atinjam direta ou indiretamente um público amplo, principalmente a população com baixa escolaridade e baixo nível socioeconômico.

Palavras-chave: Infecção sexualmente transmissível; População; Educação em saúde.

\begin{abstract}
Objective: To report an experience lived by the authors of the study in a waiting room of an Ambulatory in Belém do Pará, regarding the relationship of citizens' perception of Sexually Transmitted Infections. Method: Explains an experience report, carried out by Nursing and Medical students at the Specialty Clinic for users who were expecting care. The construction of the activity was based on the stages of the Arco de Maguerez: I- It was the elaboration of the activity in the Outpatient Clinic; IIIdentification of key points, possible vulnerabilities, and target audience; III- Theorization, research through bibliographic studies; IV-Search for technological innovations related to the problem, and construction of the material, highlighting the main STIs; V- Carrying out the educational action. Results and Discussion: The educational activity provided important dialogues about STI knowledge. Fragility was perceived in terms of knowledge and, therefore, greater interest in the transmission of
\end{abstract}


STIs. Final Considerations: It is concluded that it is fundamental to develop strategies aimed at public health, with a focus on prevention, such as the inclusion of actions aimed at qualifying knowledge about STIs, using tactics that directly or indirectly reach a wide audience, especially the population with low education and low socioeconomic status.

Keywords: Sexually transmitted infection; Population; Health education.

\section{Resumen}

Objetivo: Objetivo: Informar una experiencia vivida por los autores del estudio en una sala de espera de un Ambulatorio en Belém do Pará, relación de la percepción de los ciudadanos sobre las infecciones de transmisión sexual. Método: Explica un informe de experiencia, realizado por estudiantes de Enfermería y Medicina de la Clínica de Especialidades para usuarios que esperaban atención. La construcción de la actividad se basó en las etapas del Arco de Maguerez: I- Fue la elaboración de la actividad en la Clínica Ambulatoria; II- Identificación de puntos clave, posibles vulnerabilidades y público objetivo; IIITeorización, investigación a través de estudios bibliográficos; IV- Búsqueda de innovaciones tecnológicas relacionadas con la problemática y construcción del material, destacando las principales ITS; V- Realización de la acción educativa. Resultados y Discusión: La actividad educativa brindó importantes diálogos sobre el conocimiento de las ITS. Se percibió fragilidad en cuanto al conocimiento y, por tanto, mayor interés en la transmisión de ITS. Consideraciones finales: Se concluye que es fundamental desarrollar estrategias dirigidas a la salud pública, con foco en la prevención, como la inclusión de acciones encaminadas a calificar el conocimiento sobre las ITS, utilizando tácticas que lleguen directa o indirectamente a un amplio público, especialmente población con bajo nivel educativo y nivel socioeconómico bajo.

Palabras clave: Infección de transmisión sexual; Población; Educación para la salud.

\section{Introduçãa}

As Infecções Sexualmente Transmissíveis (IST) podem ser disseminada mediante o contato sexual (oral, vaginal, anal) sem a prática da utilização do preservativo masculino ou feminino com pessoas já infectadas. Essa infecção pode ser por vírus, bactérias ou outros microrganismos. Pode, ainda, ocorrer a transmissão por meio vertical, ou seja, da mãe para a criança durante a gestação, parto ou amamentação (Brasil, 2017).

As IST's são consideradas um grave problema de saúde social, visto que, encontram-se entre as razões mais comuns de doença no mundo e têm, em muitos territórios, vastas repercussões de natureza sanitária, coletiva e econômica. O diagnóstico precoce das IST's pode ter como resultado complicações e sequelas graves, incluindo infertilidade, perda fetal, gravidez ectópica, cancro anogenital e morte prematura, bem como infecções em recém-nascidos e lactentes (Pinto; Basso; Barros \& Gutierrez, 2018).

Dessa forma, cabe ressaltar que dentre as IST's existentes somente HIV/aids, sífilis e hepatites são de notificações compulsórias. Acredita-se há existência de muitas IST subnotificadas, fato que preocupa as autoridades sanitárias do país, considerando a sexualidade um assunto pouco debatido nos grupos sociais, cercado de mitos e tabus (Spindola et al, 2015).

A Organização Mundial da Saúde (OMS), expõe, com frequência, diversas estimativas da extensão e seriedade destas infecções de maneira global, para contribuir com a efetuação de políticas públicas para o controle. Desse modo, estima-se que 530 milhões de pessoas estão infectadas pelo vírus do herpes genital e milhares de mulheres estão infectadas pelo papilomavírus humano (HPV). Dado que, o contágio pelo HPV causa 530 mil casos de câncer de colo uterino e 275 mil mortes anualmente e a sífilis gestacional causa, aproximadamente, 300 mil mortes fetais e neonatais por ano e coloca 215 mil recémnascidos sob o risco de morte prematura, baixo peso ao nascimento ou sífilis congênita (Baião, 2018).

Sendo assim, a predominância da IST possui uma avaliação e compreensão complexa, devido à delicadeza e desapropriação dos conjuntos de vigilância. No Brasil, a situação epidemiológica das IST’s não é bem entendida, em razão da maioria das doenças não serem de notificação compulsória, além da ausência de conteúdos e de bases populacionais (Pinto; Basso; Barros \& Gutierrez, 2018).

A carência de acesso nos setores de saúde retrata no crescimento impulsivo das ISTs, e em muitos continentes essas infecções podem configurar grandes perdas econômicas, sendo elas provocadas pelos aspectos de saúde e doença. Assim, as IST representam a segunda maior causa de morbidade em mulheres juvenis adultas, nos lugares em desenvolvimento. Estima- 
se que o número de pessoas acometidas por IST curáveis no mundo ao ano seja aproximadamente 340 milhões (Pinto; Basso; Barros \& Gutierrez, 2018).

Segundo Andrade et al (2017), a fragilidade está pontualmente associada ao nível e particularidade das informações e conhecimentos que os usuários possuem de determinado problema e das possibilidades de enfrentá-la. Consideram-se fatores pessoais, como o grau de entendimento, escolaridade e acesso à informação; subjetivos, incluindo valores e crenças; biológicos, comportamentais e afetivos, que implicam em exposição e suscetibilidade ao agravo.

Desse modo, a vulnerabilidade social é desenvolvida por intermédio de informações, saúde, educação, cultura e emprego, além da prática de mudança, ao obter uma nova informação, situações estas que estão interligadas ao acesso a recursos materiais e equipamentos sociais. A vulnerabilidade programática caracteriza-se pelo reconhecimento e investigação de cenários de programas governamentais, incluindo a políticas, programas, serviços e ações de proteção e promoção à saúde (Andrade et al, 2017).

Spindola et al (2015), percebeu que o crescente número de IST ocorreu em indivíduos em que estão inseridos em uma elevada vulnerabilidade social, e não utilizam métodos de preservativos para proteção. Com isso, interfere na compreensão de questões socialmente relevantes como as maneiras de contágio e profilaxia das infecções.

Ademais, é necessário obter estratégias de prevenção das IST's e divulgar sobre os possíveis modos de transmissão e sinais/ sintomas, com o propósito de elevar o entendimento da população e orientar à procura antecipada por assistência. Um estudo retratou que, a escola é o lugar onde os jovens deveriam preferencialmente obter as informações e orientações sobre IST. Em consenso com outra literatura, observa-se que os materiais retratados pelas mídias sociais sobre IST/aids ainda são insuficientes. Além disso, mesmo recebendo tais informações os jovens ainda falham em aderir medidas de prevenção e proteção contra as IST's (Pinto; Basso; Barros \& Gutierrez, 2018).

Dessa forma, diante do exposto, tem-se como objetivo geral, relatar uma experiência educativa em Ambulatório em Belém do Pará, sobre o conhecimento acerca das Infecções Sexualmente Transmissíveis.

\section{Metodologia}

Explana um estudo qualitativo, descritivo, tipo relato de experiência, realizado por acadêmicos de Enfermagem e Medicina, nos quais são os integrantes projeto de extensão no ambulatório de especialidade de uma instituição de ensino superior (IES) privada, localizada na cidade de Belém (PA), realizado no mês de fevereiro de 2020.

O projeto de extensão "Cuidado interdisciplinar de atenção integral à saúde da mulher" visa desenvolver ações e atividades de educação em saúde, com objetivo de garantir o atendimento à mulher em sua integralidade, terciando os diversos aspectos que permeiam no atendimento ambulatorial em todas suas complexidades e almejando o atendimento integral da paciente. Nessa perspectiva, é fundamental considerar a atividade de educação em saúde entre mulheres e par sexuais, no fortalecimento de conhecimento, e ações de cuidado a saúde reprodutiva e sexual.

Dessa maneira, tivemos como público-alvo em média, de 20 usuários de ambos os sexos no qual estavam na sala de espera aguardando consultas no ambulatório de especialidade de uma IES privada, na cidade de Belém.

A construção da atividade foi baseada na metodologia da problematização e das etapas do Arco de Maguerez, as quais: I- Foi a elaboração da atividade no Ambulatório onde o projeto é desenvolvido; II- Identificação dos pontos-chaves, ou seja, possíveis vulnerabilidades e público alvo que iriamos realizar a sala de espera; III- A teorização, onde ocorre a investigação através da imersão teórica, ou seja, buscou-se nas literaturas estudos bibliográficos que oferecessem o embasamento cientifico a respeito do tema e que abordassem a prevenção das IST; IV- Busca inovações tecnológicas referentes à problemática, através de metodologias ativas que busca minimizar a situação identificada, por meio disso a construção do material, evidenciando as principais ISTs; e V- Realização da ação educativa na sala de espera para indivíduos 
que estavam no aguardo do atendimento no ambulatório de especialidades.

\section{Resultados e Discussão}

A atividade educativa foi desenvolvida por intermédio de discussões sobre as principais ISTs, como a sífilis, vírus da imunodeficiência humana (HIV/AIDS), gonorréia e Papilomavírus Humano (HPV). Na explanação da temática foram utilizadas imagens sobre as causas, consequências e prevenção das IST's a fim de contribuir para melhor compreensão no processo de ensino e aprendizado.

Durante a discussão, o público realizou alguns questionamentos sobre a transmissão das IST's, como: "é possível pegar AIDS compartilhando vaso sanitário?"; "se pega alguma IST dividindo talheres e copos?" e "pega HIV no abraço e no beijo?".

Assim, compreendem-se que houve participação efetiva das pessoas durante a atividade educativa, no entanto, percebemos significativos questionamentos direcionados para as formas de contágio, não havendo maior destaque dos participantes para a prevenção especificamente. Nota-se, desta maneira, que a preocupação da população ainda se resume nos fatores de transmissão e há um certo desinteresse nos fatores de prevenção. Destaca-se também na exposição de dúvidas, a fragilidade do conhecimento sobre os modos de transmissão e de maneira geral sobre as IST, percebido pela verbalização da dúvida da transmissão de ist pelo uso de talheres e ou compartilhamento de vaso sanitário.

Após o término da apresentação, observou-se que os usuários ainda têm uma grande fragilidade no processo de compreensão acerca da importância da prevenção para redução dos eventos de IST (Pinto; Basso; Barros \& Gutierrez, 2018).

Nota-se, que a prevenção parece ser mais trabalhosa e desconfortável para os cidadãos, visto que a principal forma de precaução das IST's é através do uso do preservativo, pois observou-se uma certa resistência dos clientes quanto aos fatores de prevenção, pois está principalmente relacionada a sexualidade (Santos \& Assis, 2011).

Além disso, identificamos que ainda existe um paradigma quando se fala em sexualidade. Spindola et al (2015), evidencia que a ausência de diálogo entre pais e filhos, educadores e trabalhadores de saúde, contribui para a eventualidade de agravos na saúde sexual/ reprodutiva dos jovens, inclusive com manifestação de IST.

Colocar um texto introdutório sobre a importância da atividade educativa nos espaços de producao de saúde. Carvalho; Pinto e Santos (2018), contribuíram que a participação dos profissionais da saúde na disseminação do conhecimento acerca das IST's é essencial. Nesse sentido, o termo humanização ganha cada vez mais espaço no âmbito das práticas em saúde.

Ademais, segundo Santos e Assis (2011), asseguram que as campanhas de ISTs são equivalentes e necessárias para grande parte da população-comunidade, as modificações nos costumes e práticas são de ficitárias, necessitando de supremas interferências culturais nesses aspectos. Bem como, no estudo de Pimenta et al (2014), em que dá destaque e relevância em maiores investimentos campanhas sobre IST, com amplitude em toda a população, e em inúmeras épocas, com objetivo nas variadas doenças.

Dessa forma, é necessário a intensificação do processo de ensino e aprendizagem acerca das IST's nos espaços formais e informais para o progresso da saúde a população, com intuito de propiciar maior empoderamento dos usuários e tomada de decisão na adoção de ações de promoção da saúde.

\section{Considerações Finais}

Conclui-se que, é uma temática que deveria ser ainda mais debatida, para que, assim, quebre essa barreira em razão dos mitos e tabus. Entretanto, ainda perfaz um enorme déficit no conhecimento a respeito das IST's e que, muitas vezes, há 
pouca qualificação do que se sabe, favorecendo, assim, muito além dessas percepções errôneas, ações com risco potencial à saúde, incluindo a do parceiro.

Ademais, a ocasião de IST's é uma realidade em qualquer faixa etária, porém tem maior prevalência na população jovem, o que acaba sendo um problema de saúde pública, no qual necessita da atenção e intervenção da equipe de saúde. Assim, as operações de educação à saúde e sensibilização da população acerca das IST's e meios para a sua prevenção, devem ser estimuladas pela articulação entre familiares, educadores e trabalhadores de saúde.

É necessário o prosseguimento de ferramentas voltadas à saúde pública, com perspectiva na prevenção e nas barreiras de agravos, como a inserção de ações objetivando qualificar o conhecimento sobre IST, utilizando táticas que originam tanto o interesse pelo tema quanto atinjam direta ou indiretamente um público amplo, principalmente os usuários com baixa escolaridade e baixo nível socioeconômico.

Dessa forma, a educação em saúde é de suma importância para comunidade sempre visando a qualidade de vida do indivíduo, que se dá pelo processo de ensino-aprendizagem, onde busca haver trocas de saberes e experiencias entre serviçocomunidade a fim de possibilitar importantes reflexões sobre o cuidar em saúde.

\section{Referências}

Abiodun, O., Sotunsa, J., Ani, F., Jaiyesimi, E. (2014). Knowledge of HIV/AIDS and predictors of uptake of HIV counseling and testing among university student students of a privately owned university in Nigeria. BMC Res Notes 7:639. https://bmcresnotes.biomedcentral.com/articles/10.1186/1756-0500-7-639. $10.1186 / 1756-0500-7-639$

Andrade, J., Ayres, J. A., Alencar, R. A., Duarte, M. T. C. \& Parada, C. M. G. L. (2017). Vulnerabilidade de idosos a infecções sexualmente transmissíveis. Acta paul. enferm. São Paulo, 30(1): 8-15. 10.1590/1982-0194201700003

Baião, A. (2018). Intervenção educativa na prevenção de doenças sexualmente transmissíveis em jovens de 18 a 29 anos. Trabalho de Conclusão de CursoCurso de Especialização Gestão do Cuidado Saúde da Família, Universidade Federal de Minas Gerais, Cruzeiro do sul /Acre.

Brasil. Ministério da Saúde (MS). Manual de Controle das Doenças Sexualmente Transmissíveis. Brasília: MS; 2006.

Brasil. Ministério da Saúde. (2017). O que são IST. http://www.aids.gov.br/pt-br/publico-geral/o-que-sao-ist

Brasil. Ministério da Saúde. (2020). Secretaria de Vigilância em Saúde. Departamento de Doenças de Condições Crônicas e Infecções Sexualmente Transmissíveis. Protocolo Clínico e Diretrizes Terapêuticas para Atenção Integral às Pessoas com Infecções Sexualmente Transmissíveis (IST)/Ministério da Saúde, Secretaria de Vigilância em Saúde, Departamento de Doenças de Condições Crônicas e Infecções Sexualmente Transmissíveis. - Brasília: Ministério da Saúde.

Carvalho, G. R. O., Pinto, R. G. S. \& Santos, M. S. (2018). Conhecimento sobre as infecções sexualmente transmissíveis por estudantes adolescentes de escolas públicas. Adolesc. Saude, Rio de Janeiro, 15(1), 7-17.

Carvalho, F. F.de, Takeda, E., Chagas, E. F. B., Pinheiro, O. L. (2020). Conhecimento da população privada de liberdade sobre infecções sexualmente transmissíveis. Rev. Gaúcha Enferm. 41(08): 1-9. 10.1590/1983-1447.2020.20190268

Costa, C. M. (2017). A Importância da Orientação de Jovens Escolares Quanto à Prevenção De Ist/Aids no Município de Macau/RN. Trabalho de Conclusão de Curso

Castro, E. L., Caldas, T. A., Morcillo, A. M., Pereira, E. M. A., Velho, P. E. N. F. (2016). O conhecimento e o ensino sobre doenças sexualmente transmissíveis entre universitários. Ciênc Saúde Coletiva. Available from: http://www.scielo.br/pdf/csc/v21n6/1413-8123-csc-21-06-1975.pdf. 10.1590/141381232015216.00492015

Ferreira, L. S., Silva, M. G. B. da. (2020). Abordagem na educação sexual de adolescentes em ambiente escolar: relato de experiência. Revista Textura, 14(1): 65-74. 10.22479/texturav14n1p65-74

Miranda, A. E., Ribeiro, D., Rezende, E. F., Pereira, G. F. M., Pinto, V. M., Saraceni, V. (2013). Associação de conhecimento sobre DST e grau de escolaridade entre conscritos em alistamento ao Exército Brasileiro, Brasil. Ciênc. Saúde Coletiva, 18(2), 489-497. 10.1590/S1413-81232013000200020.

Miranda, A. E, Ribeiro, D., Rezende, E. F., Pereira, G. F. M., Pinto, V. M., Saraceni, V. (2007). Associação de conhecimento sobre DST e grau de escolaridade entre conscritos em alistamento ao exército brasileiro, Brasil. Cien Saude Colet; 18(2):489-497.

Pimenta, A. T. M., Melli, P. P. S., Duarte, G., Quintana, S. M. (2014). Conhecimento de mulheres sobre alguns aspectos do papiloma vírus humano. Revista de Medicina (Ribeirão Preto). 47(2), 143-148.

Pinto, V. M., Basso, C. R., Barros, C. R. S., Gutierrez, E. B. (2018). Fatores associados às infecções sexualmente transmissíveis: inquérito populacional no município de São Paulo, Brasil. Ciência \& Saúde Coletiva, 23(7), 2423-2432. 
Research, Society and Development, v. 10, n. 4, e15910413923, 2021

(CC BY 4.0) | ISSN 2525-3409 | DOI: http://dx.doi.org/10.33448/rsd-v10i4.13923

Pinto, V. M., Basso, C. R., Barros, C. R. dos S., Gutierrez, E. B. (2018). Atores associados às infecções sexualmente transmissíveis: inquérito populacional no município de São Paulo, Brasil. Ciênc. saúde colet. 23(7): 2423-32. 10.1590/1413-81232018237.20602016

Russo, K., Arreguy, M. E. (2015). Projeto "Saúde e Prevenção nas Escolas": percepções de professores e alunos sobre a distribuição de preservativos masculinos no ambiente escolar. Physis Revista de Saúde Coletiva, 25(2): 501-523. http://dx.doi.org/10.1590/S0103-73312015000200010

Santos, A. F. M., Assis, M. (2011). Vulnerabilidade das idosas ao HIV/Aids: despertar das políticas públicas e profissionais de saúde no contexto da atenção integral: revisão de literatura. Rev Bras Geriatr Gerontol. 14(1): 147-157.

Spindola, T., Reicherte, M. R. A. P., Soares, A. B., Queli, V. F., Motta, L. E. F. (2015). Produção de conhecimento acerca das doenças sexualmente transmissíveis na população jovem: pesquisa bibliométrica. Revista de Pesquisa Cuidado é Fundamental Online. 7(3), 3037-3049.

Sociedade Brasileira de Pediatria. (2018). Infecções Sexualmente Transmissíveis na Adolescência. Departamentos Científicos de Adolescência e Infectologia 